\title{
LA-UR- $09-01503$
}

Approved for public release;

distribution is unlimited.

Title: Radiation Detection and Situation Management by Distributed Sensor Networks

Author(s): Janette Frigo, Sean Brennan, Ernst Esch, Diana Jackson, Vinod Kulathumani, Edward Rosten, Patrick Majerus, Adam Warniment, Angela Mielke, and Michael Cai

Intended for:

SPIE Defense, Security, and Sensing 2009

Los Alamos National Laboratory, an affirmative action/equal opportunity employer, is operated by the Los Alamos National Security, LLC for the National Nuclear Security Administration of the U.S. Department of Energy under contract DE-AC52-06NA25396. By acceptance of this article, the publisher recognizes that the U.S. Government retains a nonexclusive, royalty-free license to publish or reproduce the published form of this contribution, or to allow others to do so, for U.S. Government purposes. Los Alamos National Laboratory requests that the publisher identify this article as work performed under the auspices of the U.S. Department of Energy. Los Alamos National Laboratory strongly supports academic freedom and a researcher's right to publish; as an institution, however, the Laboratory does not endorse the viewpoint of a publication or guarantee its technical correctness. 


\title{
Radiation detection and situation management by distributed sensor networks
}

\author{
Janette Frigo ${ }^{a}$, Sean Brennan ${ }^{a}$, Ernst Escha ${ }^{a}$, Diana Jackson ${ }^{a}$, Vinod Kulathumani ${ }^{b}$, Edward Rostenc, \\ Patrick Majerus ${ }^{\mathrm{a}}$, Adam Warniment ${ }^{\mathrm{a}}$, Angela Mielke ${ }^{\mathrm{a}}$, and Michael Caia \\ aLos Alamos National Laboratory, P.O. Box 1663, MS D440, Los Alamos, NM 87545; \\ bDepartment of Computer Science and Electrical Engineering, West Virginia University, P.O. Box \\ 6109, Morgantown, WV, 26506-6109; \\ cDepartment of Engineering, Cambridge University, Cambridge, CB3 OSD, England
}

\begin{abstract}
Detection of radioactive materials in an urban environment usually requires large, portal-monitor-style radiation detectors. However, this may not be a practical solution in many transport scenarios. Alternatively, a distributed sensor network (DSN) could complement portal-style detection of radiological materials through the implementation of arrays of low cost, small heterogeneous sensors with the ability to detect the presence of radioactive materials in a moving vehicle over a specific region. In this paper, we report on the use of a heterogeneous, wireless, distributed sensor network for traffic monitoring in a field demonstration. Through wireless communications, the energy spectra from different radiation detectors are combined to improve the detection confidence. In addition, the DSN exploits other sensor technologies and algorithms to provide additional information about the vehicle, such as its speed, location, class (e.g. car, truck), and license plate number. The sensors are in-situ and data is processed in real-time at each node. Relevant information from each node is sent to a base station computer which is used to assess the movement of radioactive materials.
\end{abstract}

Keywords: distributed sensor networks, radiation detection, heterogeneous sensors

\section{INTRODUCTION}

Many vehicle surveillance radiation detection applications utilize portal monitor-style radiation detectors. These detectors are extremely accurate, but are large and costly. Additionally portal monitors require the use of traffic choke points to ensure vehicles pass through the portals at slow speeds and in a single-file configuration. This approach is viable in applications where a limited number of traffic routes are to be monitored in high value areas where significant sensor cost is tolerable. In such applications personnel are readily available to investigate potential alarms immediately, thus ensuring false alarms are quickly mitigated.

In contrast there are a host of applications in remote areas where choke points do not exist and personnel are not readily available. In these instances small, dispersed, autonomous and cost effective sensor systems are needed to provide a warning of potential threats, particularly for rapid deployment. These systems, though, need to maintain extremely small false alarm rates, a task that is extremely difficult given the requirement for small, low power, radiation detection technology.

Research is ongoing at LANL developing networks of heterogeneous, low cost sensors. This approach ensures data from several types of sensors are combined to provide highly confident decisions as to the presence and type of vehicles in remote, monitored regions and the presence of radiological material. Such networks process raw data at the sensor and propagate the multi-modal information over the network, allowing timely decisions to be made remotely by monitoring personnel. Instead of relying solely on a single large radiation detector, decisions are made based on a combination of corroborating evidence from multiple sensors. In our field experiments the monitoring network employed seismic sensors to detect the presence of a vehicle, acoustic sensors to determine the type of vehicle, photo sensors to collect license plate information about the monitored vehicle, wide-area video surveillance to provide tracking 
information and radiation detectors to determine the presence of radioactive materials. Such a system has been successfully demonstrated in remote canyons within the LANL complex and has the ability to significantly alter the methods currently utilized to provide wide area persistent surveillance.

The remainder of this paper is organized as follows: Section 2 describes our approach for detection of radiological materials in moving vehicles and how it compares to that of the research community. Section 3 discusses the heterogeneous sensors nodes; the algorithms, deployed system implementation. Section 4 explains our field experiment; the communication system, base station graphical user interface (GUI), and field test setup. Finally, in Section 5 we show our system test results for the DSN and share some of our field experiences.

\section{RELATED WORK}

Simulation test beds are an important first step in any sensor network system. However, moving from the simulation test bed to hardware implementation is costly and time consuming for any deployed system whether the system is a satellite; a mobile robot or a sensor network. Most sensor network research for radiation detection is in simulation only, such as in Parunak's [2] simulation for radiation detection and situational management, a large dynamic spatio-temporal configuration of sensors are used. Others discuss the challenges and possible solutions of transportation security, including intra-modal transport venues, using knowledge discovery tools [1].

In our own previous work, the Distributed Sensor Network project at LANL looked at the feasibility of a small network of sensor nodes using PDA-sized platforms for processing raw gamma counts [3]. In [4] and [5] the feasibility of a network of detectors for measuring radioactive materials along a known trajectory and the ramifications thereof are examined. In [6] the effectiveness of scaling large numbers of nodes (greater than 10) and changing source trajectory parameters versus the computational demands required for such a task are explored.

In this paper we report our attempt to implement a deployed multi-modal sensor network for the detection of moving radioactive materials along a roadway. The goal of this work reported herein is to implement the system using low power, Commercial Off-The-Shelf (COTS) hardware and thereby evaluate its effectiveness. Our approach is to use low power, wireless sensor nodes. For detection of radioactive material, multiple radiation detectors sum spectra using coherent addition to obtain an increase in signal-to-noise compared to a single detector, thus, by increasing the number of detectors, the collected spectrum is significantly improved. We determined through experimentation that approximately 40 radiation detectors (NaI scintillators per Section Error! Reference source not found.) could give adequate coverage over a given region to detect with good fidelity. Details are provided in Sections 3.3 - Error! Reference source not found.

\section{HETEROGENEOUS SENSOR NODES}

This section describes the system configuration, algorithms, and implementation for the three types of sensor nodes in our DSN system: vehicle class identification, vehicle license plate detection, and radiation detection.

\subsection{Vehicle Class Identification}

The goal of this vehicle classifier node is to classify vehicles as they approach the region into one of three categories: a small compact car, a moderately heavy vehicle and a very heavy vehicle. For our training and testing we chose a 1994 Honda Accord LX, manual drive compact car, a 2006 Diesel Chevy C4500 4x4 truck, and a 1994 HumV H-1 with a 6.5 L Detroit Diesel Engine as representative vehicles of each category. We assume that vehicles do not enter the monitoring area concurrently. Particular challenges and assumptions for this classifier node are: (1) Vehicles travel between 10 to 40 $\mathrm{mph}$ and stay within the influence region of the sensors for a very short time of 1 to 2 seconds only; (2) The audio spectral signature of the vehicle can change over time; (3) The algorithms cannot be computationally intensive. 


\section{Vehicle classifier implementation}

The vehicle classifier node developed uses seismic and acoustic sensors connected to a Crossbow Mica2 mote and a Stargate respectively (see Figure 1). The seismic sensor is a GeoSpace geophone placed 50 feet from the road to eliminate acoustic feedback in the sensor. The geophone is connected to the Mica2 via a custom signal conditioning interface board and a 16-bit A/D board. The acoustic sensor is a Samson C01U USB Studio Condenser Microphone, placed 10-12 feet from the road and mounted 1 foot off the ground. The microphone is connected directly via a USB port to the Stargate $(400 \mathrm{MHz}$, Intel PXA255 Processor, Linux based). The microphone has directional response and is mounted facing toward the roadway. Windshields on the microphones help filter wind noise.

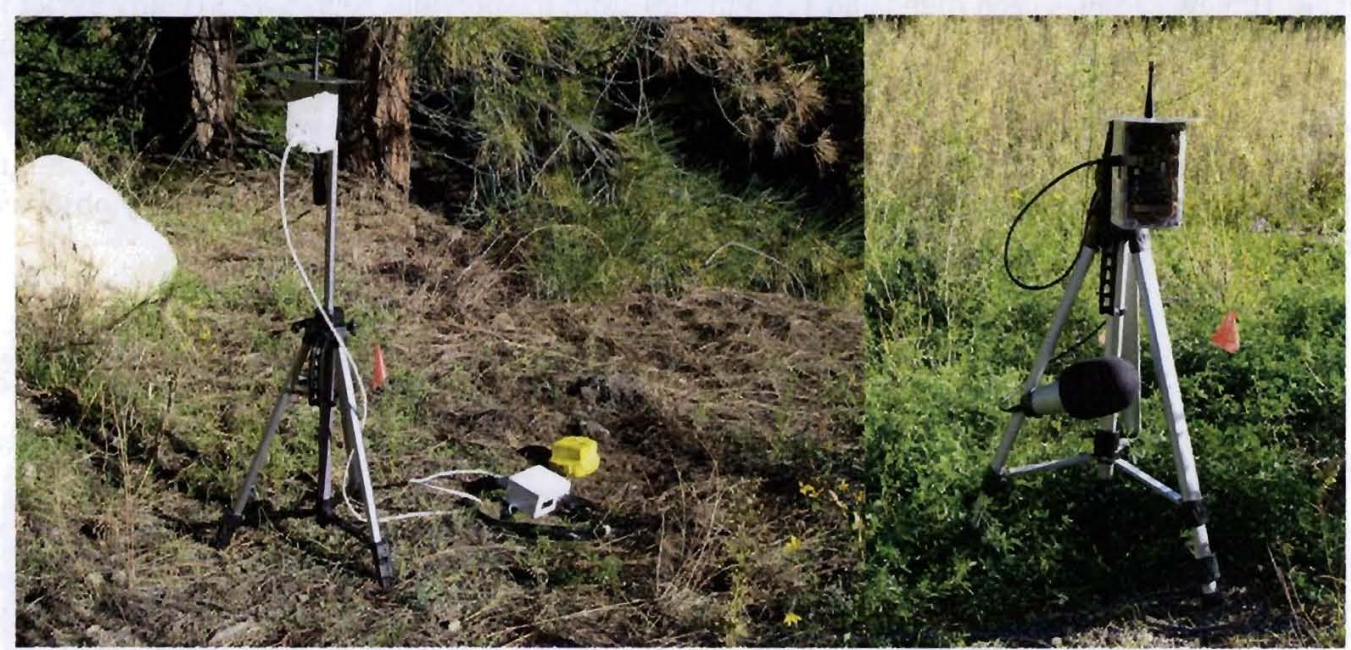

Figure 1 Mica2 mote, signal conditioning box, seismic sensor (left). Stargate and acoustic sensor (right)

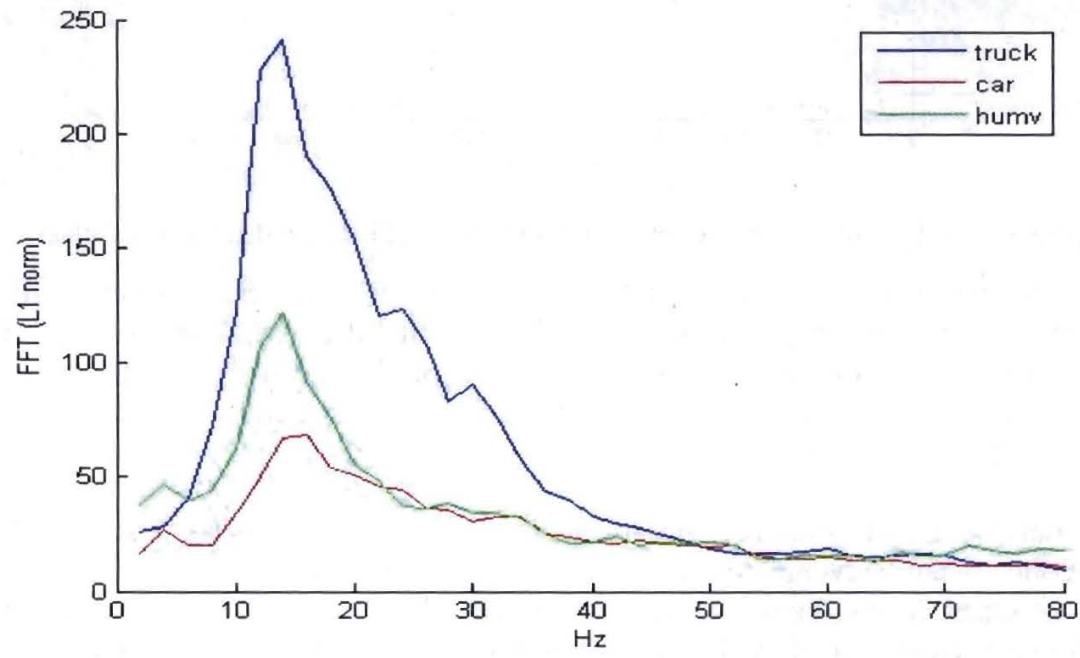

Figure 2 Frequency characteristics of seismic detection

This implementation uses the Mica2 mote to trigger an event based on seismic information. The event is transmitted to the Stargate processor over the $900 \mathrm{MHz}$ radio link. The Stargate then samples the microphone and processes acoustic information and sends a classification to a base station computer (via an 802.11 network). We use this method of seismic detection triggered acoustic sampling and processing because it is an energy efficient way of fusing the multi-sensor information to yield a single classification, i.e. the seismic detection runs continuously at approximately 60 milliWatts compared to the Stargate/acoustic processing which takes about 2.4 Watts. Moreover, the frequency characteristics for seismic detection show very similar peak frequencies (see Figure 2) so more frequency analysis would have to be done 
in order to develop an accurate classification using seismic data and the Mica2 does not have sufficient computing capabilities to do this. For these reasons, we choose to combine both seismic and acoustic sensors to achieve a more reliable, energy-efficient classification.

\section{Vehicle classifier algorithms}

The geophone is sampled at $100 \mathrm{~Hz}$. The Mica 2 mote computes the Haar Wavelet on a moving window of 128 samples every $10 \mathrm{~ms}$ using 10 new samples each round and 118 samples from the previous round. The Haar wavelet is computed up to level 2 which computes the energy estimate of the $12-24 \mathrm{~Hz}$ band via the average of the coefficients of this band. The variance of the energy estimate is computed on a moving window of size 20 (see Figure 3). A variance threshold is used for vehicle event detection. A trigger is sent to the Stargate over the radio link when a vehicle event occurs. The Haar Wavelet is chosen for it's low-level of computational complexity which was required due to the 8-bit computing capability of the ATMEL processor on the Mica2 [7] and also because of the narrow peak frequency observed for all the vehicle categories.

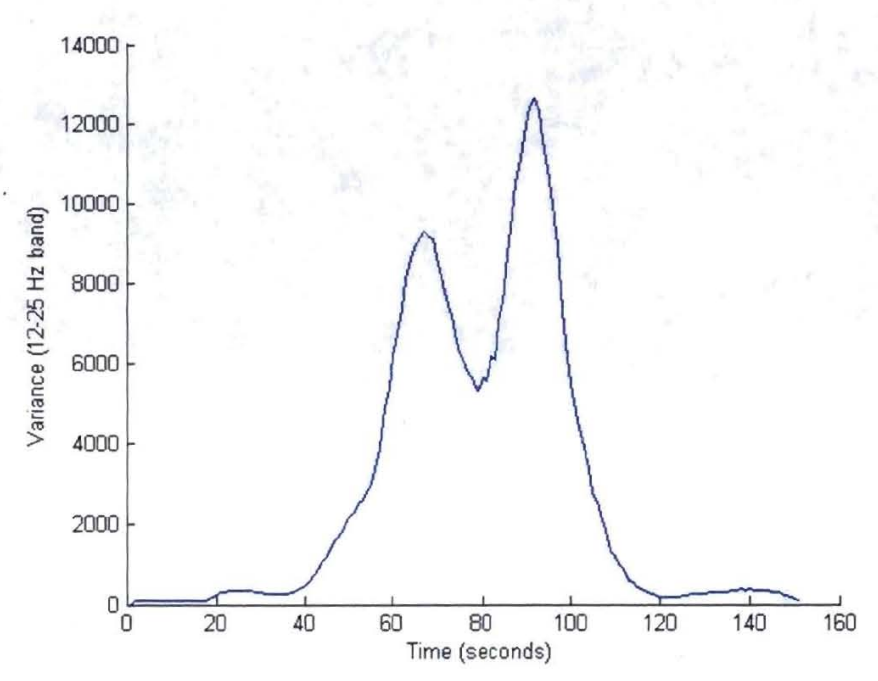

Figure 3 Moving window variance characteristics of $12-25 \mathrm{~Hz}$ band for a 'truck' class

Upon receiving a trigger, the microphone is sampled at $4 \mathrm{kHz}$. There are four sources of sound collected by the acoustic sensor, i.e. road/tire, engine, mechanical and air current noise. For the classifier, a 512-point integer FFT is implemented on the Stargate. The 512-point FFT is computed every $125 \mathrm{~ms}$ to obtain the spectral characteristics of the data, yielding an $8 \mathrm{~Hz}$ resolution. Frequencies lower than $64 \mathrm{~Hz}$ are not used due to variations in the microphone response and temporal variations (wind) at these lower frequencies.

We first obtain training data sets using multiple runs through each vehicle at different speeds. We use the samples collected during the 2 seconds when the vehicle is closest to the microphone for the training. We then identify the ideal feature vector set to do the classification between each pair of vehicles. For example to classify between a car and a truck, we use a 10 coefficient vector, formed by the average energy of 10 equally spaced bands in the $224 \mathrm{~Hz}$ to $368 \mathrm{~Hz}$ range because the spectral characteristics of the truck shows a distinctive spike in response at those frequencies. We use Fisher Linear Discriminant Vector analysis to identify the best projection vector given the training data. We obtain a similar projection vector to distinguish whether the vehicle is a Humv or a car/truck. These projection vectors are computed offline in matlab and then copied into the classifier program running on the Stargate.

In every round, the Stargate simply computes the dot product of this vector with the feature vector obtained in that round to perform the classification. The Stargate first classifies whether the vehicle is a Humv or either of car or truck. Then it classifies whether the vehicles is a car or a truck. We find that this is the classification order that maximizes the distance between classes. 
Once triggered the classifier operates for 3-4 seconds as the vehicle passes the sensor (until the seismic sensor sends a signal to stop classification). The acoustic classifier is operating in real-time and generates a classification output once every $125 \mathrm{~ms}$. The characteristics of an approaching vehicle differ from when the vehicle is at the closest distance to the sensor. The central base station computer integrates the individual classification outputs to generate the final classifier output.

\section{Vehicle classifier accuracy}

We achieved no false negatives from the seismic sensor during 10 field trials. A person walking as close as 2 feet away from the sensor does not trigger an event. A person jumping less than 10 feet away from the sensor triggers a seismic detection; however this case can be isolated using temporal characteristics.

The initial work on the acoustic classifier also showed zero misclassification's in 10 test runs of each vehicle class. Note that, we maintain our testing environment to be similar to the training environment. We also ensure that vehicles enter the field one at a time. Training the classifier in a dynamic manner to different environments is a much more difficult problem and is a subject of future work.

\subsection{License Plate Detection}

The vehicle license plate detection node aims to capture the image of a vehicle traveling on the roadway, reduce the image to license plate pixels only using a learning algorithm and resize this reduced image for efficient transfer over the network to the base station. The image processing algorithm reduces the original image by 60 to $90 \%$, and allowing the image to be converted to text via an Optical Character Recognition (OCR) application on the base station.

\section{License plate node implementation}

The video sensor node consists of a webcam (with a $12 \mathrm{~mm}$ telephoto lens) and a Honeywell magnetometer (HMR2300232) connected directly to the Stargate via the USB port and the serial port respectively. The magnetometer is used as a trigger for image capture. A learning algorithm converts the original image to license plate pixels only. This processed image information is sent over the $2 \mathrm{GHz}$ wireless link to the network. The sensors and Stargate are mounted on a tripod located approximately 10 feet away from the road and 3 to 4 feet off the ground. To eliminate glare, the assembly is slanted at about a 45 degree angle to the road.

The system requirements are as follows: to capture a $640 \times 480$ pixel image of the aft end of a vehicle at anticipated vehicle speeds of 10 to $60 \mathrm{mph}$, to extract the license plate pixels only from the original image, thereby reducing the original image by approximately $60-90 \%$.

Figure 4 shows a vehicle image captured from the webcam. We chose to use the webcam due to its ease of integration, low power, low cost and compact size. In addition, the image resolution with our telephoto lens was sufficient for the learning algorithm. The best range for image capture is within 8 to 15 feet from the camera. The magnetometer triggers on the front end of the vehicle and we capture images for 5 seconds at approximately 10 frames/sec. From this set of frames, on original image is chosen to be processed.

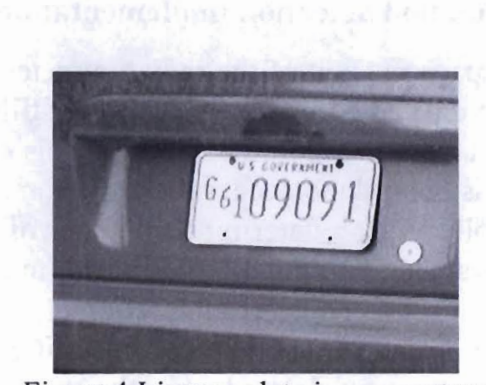

Figure 4 License plate image capture 


\section{License plate detection algorithms}

The processing on the license plate detection node works by applying a classifier to every pixel in an image to create a rough segmentation of the license place, if it exists. From this, the bounding box of the license plate is found, and that section of the image is then resampled to a fixed size. The resampled image is then PNG compressed and sent over the network. These steps are a trade off between the amount of network bandwidth used, the latency of the operation and the amount of computing power used locally.

The classifier is trained using data collected during typical field operation. This consists of various vehicles viewed at distances of between 8 and 75 feet from the camera, as well as some 'background' images containing no license plates. A eight bpp greyscale image is used for the algorithm development.

The license plate detection software has to be able to process full video images on the Stargate processor, so a very efficient algorithm is required. The license plate detection is performed using a machine learning algorithm, trained on labeled data. To achieve very high speed video processing we use an algorithm which takes elements from two algorithms known to produce very efficient classifiers, namely the Viola-Jones [8] object detection algorithm and the ID3 [9] decision tree classifier. The algorithm details for license plate segmentation, bounding and resampling are found in [10]. Figure 5 shows a sample output from the license plate detection node.

Accuracy measurements for the license plate detection node were taken with two vehicles: a compact car, and a 2008 Diesel $4 \times 4$ pickup truck. We estimate license plate identification accuracy to be about $90 \%$ based on field trials with the set of two training vehicles. The OCR application results from license plate image are a topic of further research.

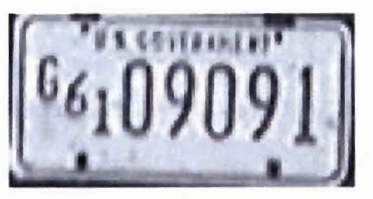

Figure 5 License plate detection output image

\subsection{Radiation Detection}

Whereas our other sensing nodes could operate in a rather loosely-coupled fashion to arrive at conclusions, our radiation detection nodes were much more tightly coupled. Our hypothesis - that multiple small detectors working in concert can be substituted for one large detector - in fact tequires this strong cooperation. We equipped two Stargate nodes with Amptek GAMMA-RAD $76 \times 76 \mathrm{~mm}\left(3^{\prime \prime}\right) \mathrm{NaI}$ scintillators, and a third with an Amptek GAMMA_RAD $10 \times 10 \times 40 \mathrm{~cm}$ (4" x 4" x 16") NaI detector. These three nodes collected background and triggered-signal data and transmitted that data wirelessly to a fourth node which combined all this data into a single spectrum for examination. For background calibration purposes a small bag of potassium-chloride was placed next to the detectors.

\section{Radiation detection implementation}

To distinguish when a vehicle occupies the space in front of the radiation detector, we use a Honeywell magnetometer to trigger the node. Each node is setup to take data in form of a ring buffer filled with radiation spectra. Each spectrum displays the gamma-ray energies present for a certain amount of time $t$. I this case this time was three seconds. Thereby, by generating a ring buffer with $n$ spectra, it is possible to access the radiation response for a time $t^{*} n$ in the past after the trigger occurred. Upon a trigger, the node collects a predetermined number of spectra as radiation signal. In the demo 3 spectra were taken. Also, a background, extracted from the circular buffer at a previous time ( 21 seconds), is computed by summing a certain amount of background spectra. To keep subtraction errors low, the background sum was composed of 50 spectra and then scaled to the radiation spectra time. The two resulting spectra, radiation signal and background, where sent to a collection hub. The signal spectra were deleted from the ring buffer and thereby the detector is ready for a new trigger. 
At the collection hub the data was received and the difference between the radiation event spectrum and the background spectrum was calculated. The background spectra from each Stargate were separately analyzed for the peak position of the potassium-chloride peak at $1461 \mathrm{keV}$. The resulting data calibration point was used for energy calibration each time a trigger occurred. This algorithm is necessary to prevent energy calibration drift in sodium-iodide detectors (see Figure 6) [11]. Our target radioisotope was Cesium-137 $\left({ }^{137} \mathrm{Cs}\right)$ with an activity of 300 microcuries, mounted on the near side of the bed of the Chevy $4 \times 4$ mentioned above.

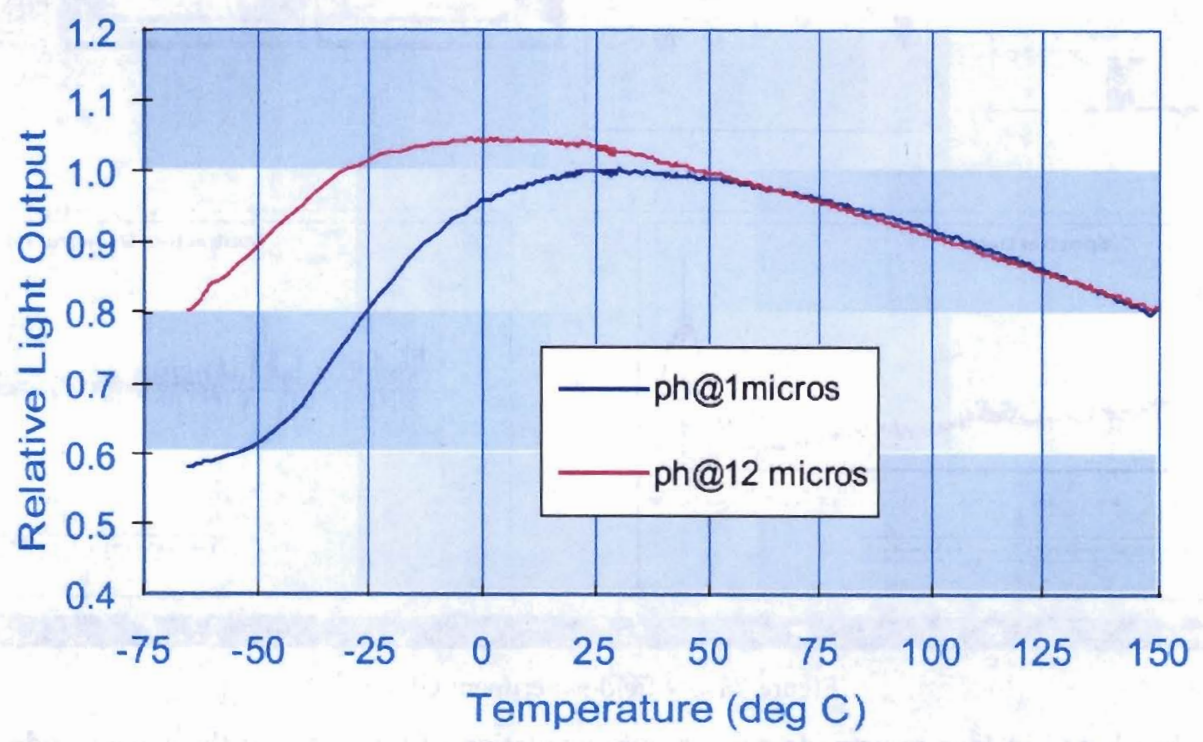

Figure 6 Nai Temperature dependant light output for $\mathrm{NaI}(\mathrm{Tl})$ chrystals [11].

Once the data calibration was done to each of the three spectra, each spectrum was filled into a new spectrum with a $1 \mathrm{keV}$ bin width. The filling process was done by an algorithm that calculated the overlap of the old spectrum bin with the new spectrum bin. The old bin value was multiplied by overlap to bin-width ratio and filled into the new bin. Once this process was done, the spectra from the different $\mathrm{NaI}(\mathrm{Tl})$ detectors could be added into a sum-spectrum. This sumspectrum now displays the radiationm coming from the suspect vehicle added over 3 detectors.

\section{FIELD EXPERIMENT}

In this section, we describe the overall DSN system layout for our field experiment including the sensor and base station nodes, the network communication, and our test scenarios.

\subsection{Network communication}

We use direct 802.11 connections from each node to the base station computer. Initially, our first attempt used several hoping nodes between the sensor nodes and the base station. However, due to the hot outdoor temperatures our 802.11 cards failed intermittently. As well, issues arose with our custom message passing software and we decided on a direct connection communicate between the sensor nodes and the base station.

\subsection{Base Station GUI}




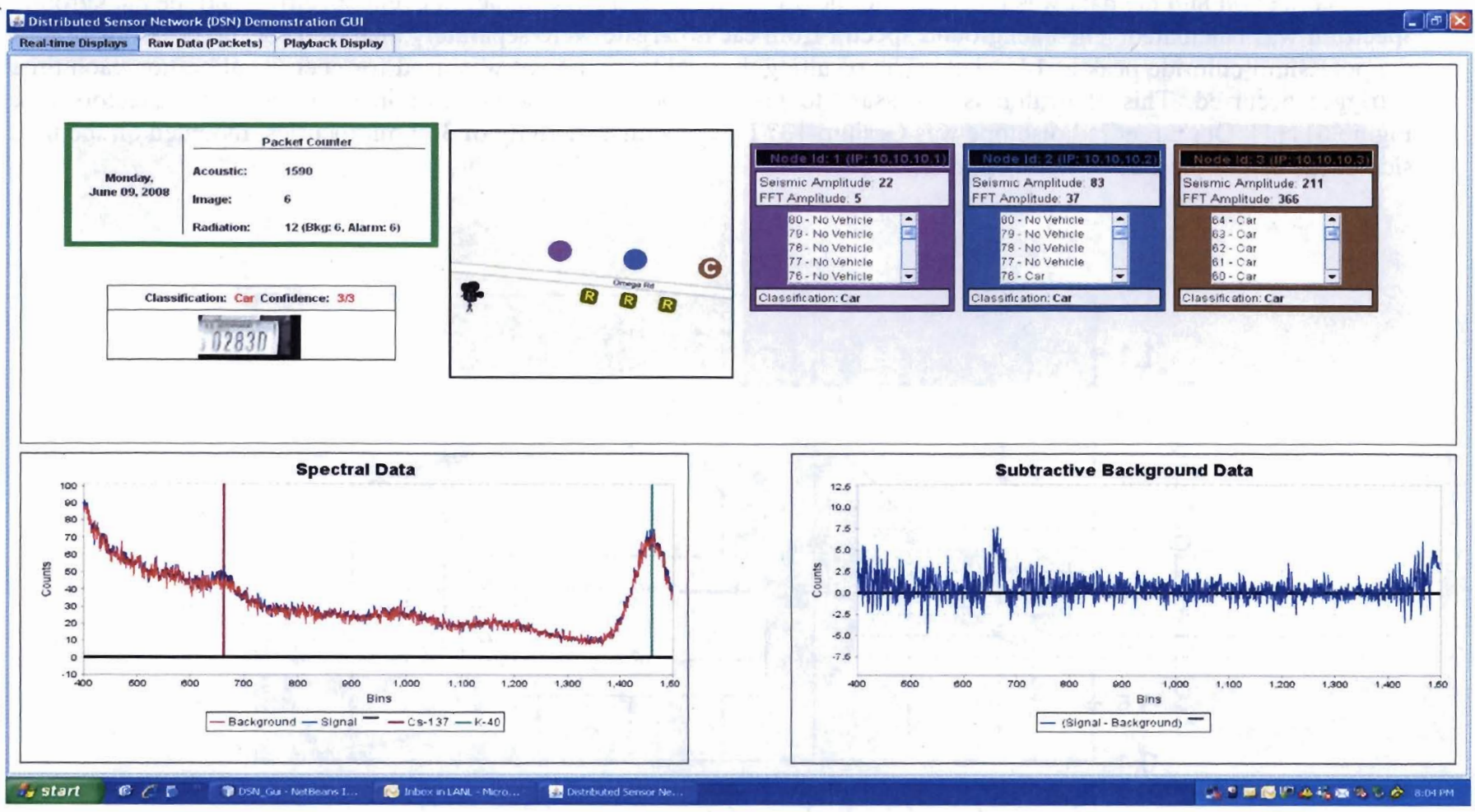

Figure 7 DSN field experiment GUI

The DSN Gui was developed in Java to provide a visual representation of the status of the sensor nodes within the DSN network. The GUI operates in one of two modes: playback and real-time. In real-time mode, the gui receives data packets from the radiation sensor nodes, vehicle classifier nodes, and video node.

The GUI receives real-time data from each sensor node. For the radiation nodes, the background spectra, the detected source spectra and sigma level are received as the sum of the three detector nodes-all processing is done on the nodes. The spectral data display shows the spectrum for the (signal minus background) in counts (see Figure 7). The vehicle classifier node sends a stream of classifications as the vehicle approaches and passes the seismic/acoustic sensors. An algorithm for vehicle classification processes this stream of data and determines a vehicle type for each node. Then a confidence level out of the possible number of nodes is displayed. The video node sends one png image for display of license plate pixels only. The OCR application converts the image to text. This final step, automatic conversion to text, is forthcoming, but the intension is to be able to check a data-base of allowable license plate numbers and the time to day to verify traffic activity. Finally, the GPS location of each node and the system clock is used to derive vehicle speed.

The base station GUI is essential for situational management as it allows monitoring personnel to get an immediate analysis of the spectral data in comparison with the background data to help determine the presence of a radioactive material. In addition, it also provided a real-time display of sensor nodes to determine vehicle class, license plate, and vehicle speed (not displayed in Figure 7) to aid in verifying normal and suspicious traffic movement.

\subsection{Test System Configuration}

Figure 8 shows the DSN system layout for our field experiment. Our field demonstration included seven sensor nodes; three vehicle classification nodes, three radiation detection nodes, and one license plate detection node. Sensor nodes are placed approximately 125 feet apart. Each node communicates directly via an 802.11 wireless link to the base station computer. The total distance covered on the roadway for this experiment is approximately 650 feet from end to end.

The test scenario consisted of multiple test trials per vehicle (car, truck, and HumV) traveling east bound on the roadway with varying speed of $5,10,25$, and $40 \mathrm{mph}$. A five minute rest interval between trials existed so we could check system 
readiness. Only one of the vehicles, containing the radioactive materials, was tested at speeds of 5 to $10 \mathrm{mph}$ with multiple trials.

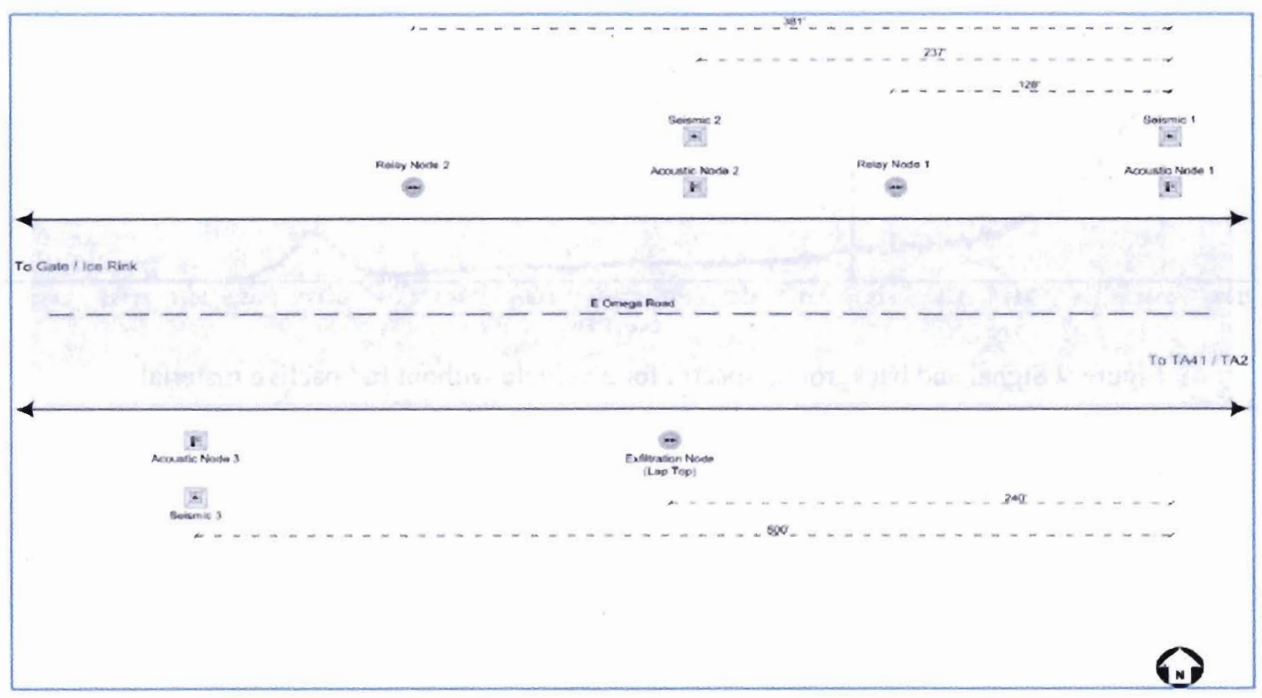

Figure 8 DSN test demonstration configuration

\section{RESULTS}

For the field demonstration the classifier performed nominally for the compact car and HumV trials at speed of 10, 25, $40 \mathrm{mph}$, each classified $100 \%$. The vehicle containing the radioactive materials drove at speeds of 5 and $10 \mathrm{mph}$ and classified as a car with a confidence of $100 \%$ since this vehicle has similar frequency characteristics to the compact car at these slow speeds.

During the field demonstration, the HumV and the car did not classify correctly on three trials. The HumV was not part of the training data for the learning algorithm and the license plate is located on the bottom left of the vehicle. The algorithm (in Section 3.7) is searching for the license plate pixels in the center of the frame between the 25th and 70th percentile horizontally and vertically. For the car, the detection was not accurate over three trails due to glare from the sun because the direction of travel was East bound in mid morning. This problem can be easily mitigated with a protective covering for the camera. The truck, however, was detected at $100 \%$ as shown in Figure XXX????????

\subsection{Radiation detection}

Figures 9 and 10 are the combined background spectrum overlapping the combined signal spectrum and the difference between the two, respectively, for a non-isotope pass. Note the drastic change in the Y-axis values between these two figures. Figures 11 and 12 correspond to the Cesium pass. The photopeaks for ${ }^{40} \mathrm{~K}$ and ${ }^{137} \mathrm{Cs}$ are marked. The Y-axes for Figures 9 and 11 and for Figures 10 and 12 are identical for clarity of comparison. Vehicles passing without a radioactive source have no peak at $662 \mathrm{keV}$ corresponding to ${ }^{137} \mathrm{Cs}$ as is just visible in Figure 11 but more obvious in Figure 12. Also note the increased counts overall for the radioisotope pass. Our calibration was somewhat imperfect in scaling, hence the peak in Figure 12 at $1461 \mathrm{KeV}\left({ }^{40} \mathrm{~K}\right)$, but the Cesium peak is very distinct. 


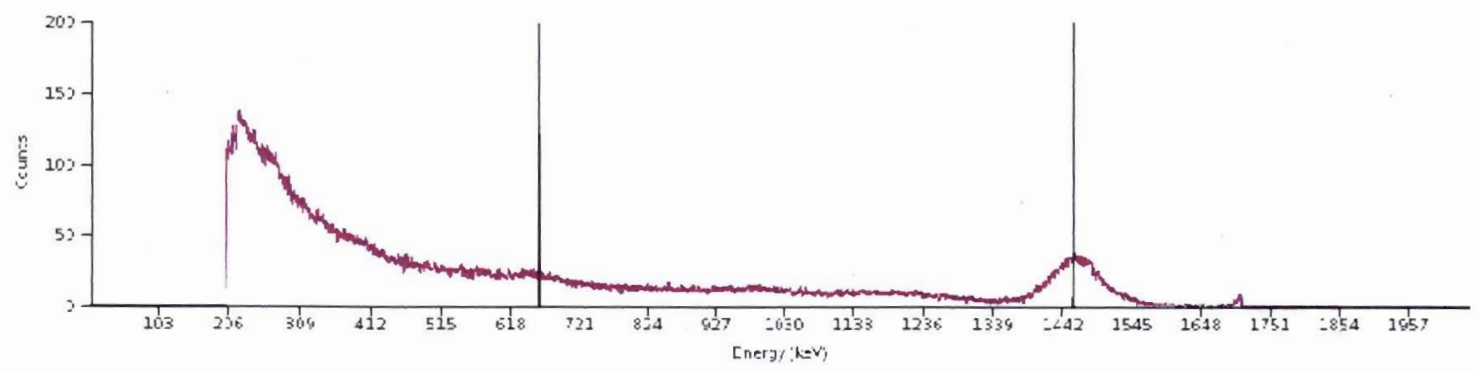

Figure 9 Signal and background spectra for a vehicle without radioactive material.

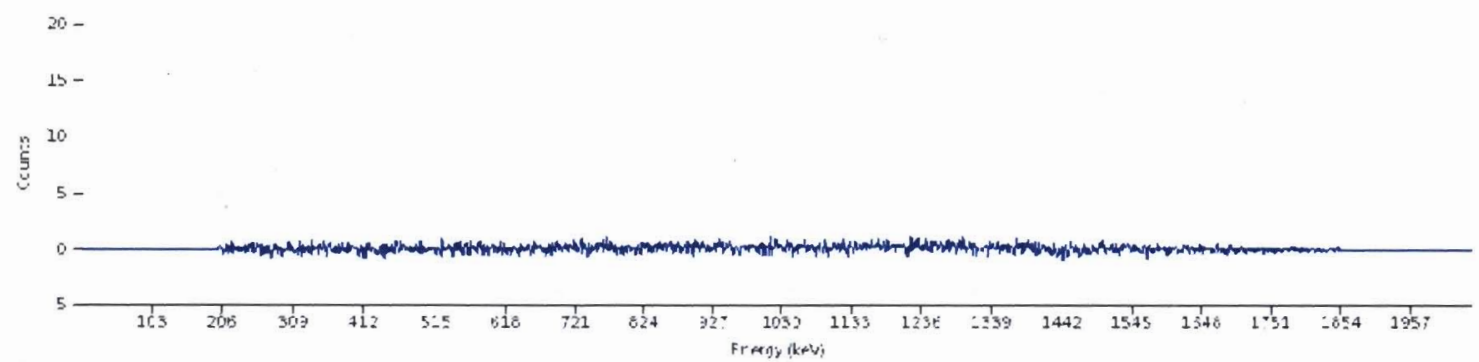

Figure 10 Difference between signal and background spectra for a vehicle without radioactive material.

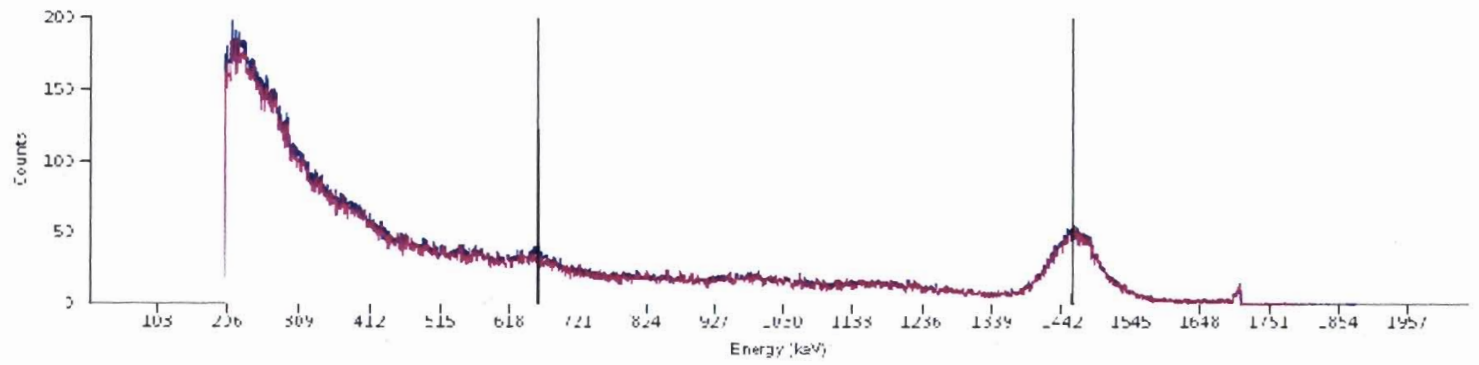

Figure 11 Signal and background spectra for a vehicle carrying ${ }^{137} \mathrm{Cs}$

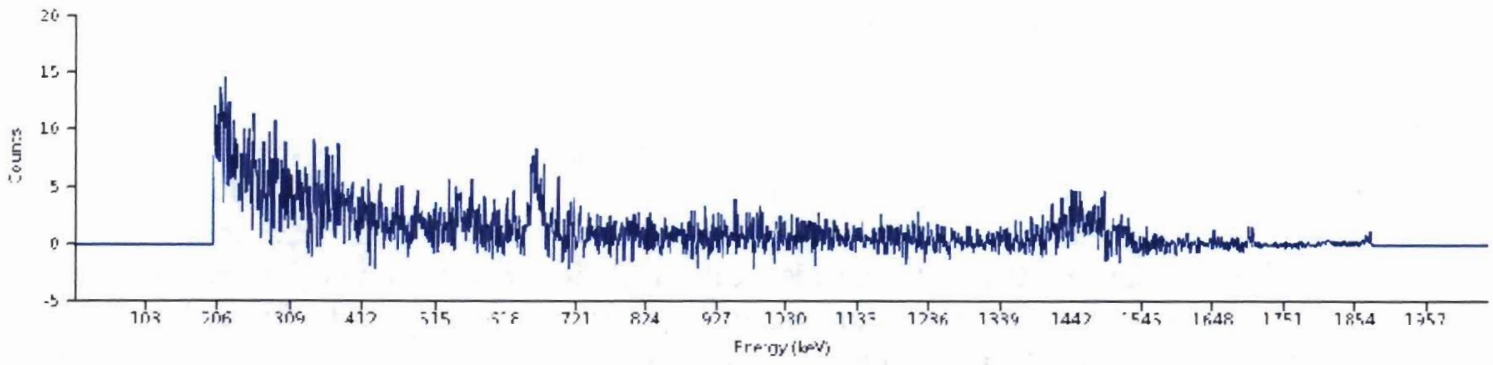

Figure 12 Difference between signal and background spectra for a vehicle carrying ${ }^{137} \mathrm{Cs}$ 


\section{SUMMARY}

A distributed sensor network may complement portal-style detection of radiological materials through the implementation of arrays of low cost, small heterogeneous sensors with the ability to detect the presence of radioactive materials in a moving vehicle over a specific region. In this paper, we report on the use of a heterogeneous wireless sensor network for traffic monitoring in a field demonstration setting. Through wireless communications, the energy spectra from different radiation detectors are combined to improve the detection confidence. In addition, the DSN exploits other sensor technologies and algorithms to provide information about the vehicle, such as its speed, location, class (e.g. car, truck), and license plate number. Multi-modal DSNs are a potential radiation detection capability, enabling deployment over a broader region without the loss of detection accuracy.

\section{ACKNOWLEDGEMENTS}

This work was supported by the U. S. Department of Energy/NNSA and Los Alamos National Laboratory funds under LANS, LLC Contract No. W-7405-ENG-36. This document is approved for public release under LAUR-09-XXXX.

\section{REFERENCES}

[1] Ganguly, A. R., et al., Knowledge Discovery from Sensor Data for Security Applications, chap 12, 187-204, 2007.

[2] Parunak, H. Van Dyke, et al., "Swarming Pattern Detection in Sensor and Robot Networks," ANS $10^{\text {th }}$ Conference on Robotics and Remote Systems for Hazardous Environments, 2004.

[3] Brennan, Sean M., et al., "Radiation Detection with Distributed Sensor Networks," IEEE Computer, Vol. 37, No. 8, Aug. 2004.

[4] Nemzek, Robert J., "Distributed Sensor Networks for Detection of Mobile Radioactive Sources," IEEE Transactions on Nuclear Science, Vol. 51, No. 4, Aug 2004.

[5] Stephens, Daniel L., et al., "Detection of Moving Radioactive Sources Using Sensor Networks," IEEE Transactions on Nuclear Science, Vol 51, No. 5, Oct 2004.

[6] Brennan, Sean M., et al., "Radioactive Source Detection by Sensor Networks," IEEE Transactions on Nuclear Science, Vol. 52, No. 3, Aug 2005.

[7] Gu, Lin, et al., "Lightweight Detection and Classification for Wireless Sensor Networks in Realistic Environments", SensSys 2005, Nov 2005.

[8] Viola, Paul A., Jones, Michael J., "Rapid Object Detection using a Boosted Cascade of Simple Features", IEEE Conference on Computer Vision and Pattern Recognition, Vol 1, pp. 511-518, 2001.

[9] Quinlan, J. Ross, "Induction of decision trees," Machine Learning, pp. 81-106, 1986.

[10] Frigo, Jan, et al., "Sensor network based vehicle classification and license plate identification system," IEEE INSS 2009.

[11] Saint Gobain Web Page: http://www.detectors. saintgobain.com/Media/Documents/S0000000000000001004/SGC_Sodium_Iodide_Data_Sheet.pdf 